\title{
Pulsed Electromagnetic Field Elicits Muscle Recovery via Increase of HSP 70 Expression after Crush Injury of Rat Skeletal Muscle
}

\author{
Songhee Cheon, PhD, PT ${ }^{1)}$, Inah Park, BSc ${ }^{2)}$, Minhee Kim, PhD, PT ${ }^{3)}$ \\ 1) Department of Physical Therapy, College of Health Science, Youngsan University \\ 2) Department of Life Science, Faculty of Art and Science, University of Toronto \\ 3) Department of Physical Therapy, College of Health Science, Eulji University: 212 Seongnam \\ Gyeonggi, 461-713 Korea. \\ TEL: +82 31-740-7206,FAX:+82 31-740-7367,E-mail:kmh12@eulji.ac.kr
}

\begin{abstract}
Purpose] The present study investigated the effects of electromagnetic field applied at different time points on muscle recovery process through observation of the expression of HSP 70 protein and histological changes in a rat muscle crush injury model. [Subjects and Methods] Fifty-four male Sprague-Dawley rats were subjected to crush injury of the left extensor digitorum longus muscle. The animals were randomly assigned to the control $(\mathrm{CON})$, pulsed electromagnetic fields (PEMF), delayed PEMF (DPEMF) groups and pulsed electromagnetic fields were administered from 1 or 3 days after muscle injury. Western blotting, immunohistochemistry, and hematoxylin-eosin staining was used to evaluate of heat shock protein (HSP) 70 expression to assess muscle recovery. [Results] Muscle injury-induced decrease of HSP 70 protein was increased and recovered by electromagnetic field application in the PEMF and DPEMF groups. Moreover, atrophy and irregular arrangement of muscle fiber caused by crush injury improved in both the PEMF and DPEMF groups. [Conclusion] In the rat model of muscle crush injury, PEMF induced muscle recovery via increased HSJP 70 protein.

Key words: Heat shock protein 70, Muscle crush injury, Pulsed electromagnetic field
\end{abstract}

(This article was submitted Jan. 16, 2012, and was accepted Feb. 22, 2012)

\section{INTRODUCTION}

Musculoskeletal impairment is the most common trauma following physical impact and is frequently seen in sports medicine 7 . Because of the diversity of musculoskeletal diseases and injuries, therapeutic approaches can range from simple analgesics to functional rehabilitation, and can result in chronic pain or loss of mobility and a severe deficit in quality of life . Despite its clinical importance, few clinical studies have reported on the treatment of musculoskeletal injury and proper therapies are needed for the restoration of muscle volume and function 1,3 .

From a clinical point of view, muscle healing involves the formation of connective scar tissue, not primarily regeneration. Especially, in the case of serious injuries or broad defects, the healing process in impaired muscle tissue is often limited and characterized by extensive muscle fibrosis creating a tendency of muscle injury to recur. For patients with delayed and imperfect posttraumatic healing process of injured muscle, regeneration enhancing strategies are required 3,4 .

Pulsed electromagnetic field (PEMF) is the one of the physical rehabilitation modalities. It is generated by external magnetic coils exciting electric fields in the conductive tissues of the body via inductive coupling including eddy current. PEMF is utilized at extremely low frequencies between 5 and $300 \mathrm{~Hz}$, but can also emphasize typically short was frequencies, such as $27 \mathrm{MHz}$. PEMF is used in many countries for a wide range of therapeutic applications and for improved well-being 6 . It has been reported that indications for magnetic field therapy include fracture healing, degenerative diseases of the musculoskeletal system, disorders of the neurological system, muscle spasm, generalized inflammation, and poor circulation ${ }^{-11}$. However, it cannot be recommended without more scientific evidence about its therapeutic effect of PEMF. Therefore, the purpose of present study was to confirm the effects of electromagnetic field applied at different time points on the muscle recovery process through observation of the expression of heat shock protein (HSP 70) protein and histological changes in a rat muscle crush injury model.

\section{SUBJECTS AND METHODS}

Fifty-four male Sprague-Dawley rats, weighing between $250 \mathrm{~g}$ and $300 \mathrm{~g}$, were used in the experiment. They were kept on a light/dark cycle with ad libitum access to food and water. All experiments were performed in accordance with protocols approved by the Animal Experiment Committee of University of Daegu, which are based on the NIH Guidelines for the Care and Use of Laboratory Animals (NIH publication, 1996). The animals were randomly divided 
into 3 groups: the control (CON), pulsed electromagnetic fields (PEMF), and delayed pulsed electromagnetic fields (DPEMF) groups. Rats were anesthetized by intraperitoneal injection with a mixture of $2 \mathrm{~mL} / \mathrm{kg} 50 \%$ zoletil and $50 \%$ xylazine hydrochloride. The left lower limb was shaved and disinfected with $70 \%$ ethanol. Through a $2 \mathrm{~cm}$ anterolateral longitudinal incision of the skins the underlying fascia from the extensor digitorum longus was mobilized. A blunt injury was induced on the left extensor digitorum muscle via an instrumented clamp (area of contact between muscle tissue and clamp: $5 \mathrm{~mm}^{2}$ ), which allowed a standardized force application of $25 \mathrm{~N}$ for $10 \mathrm{~s}$ (DMC PLUS, HBM Hottinger Baldwin Messtechnik GmbH Germany). PEMF was conducted for the PEMF and DFEMF groups. The frequency of the electromagnetic field used in the present study was 27.12 MHz, and the application time was 20 minutes; the instrument used was a Diapulse (Diapulse Corp., America). The PEMF irradiation was performed once a day for 5 days from 1 day after injury for the PEMF group, and once a day for 3 days from 3 days after injury for the DPEMF. For sacrifice, the animals were anesthetized with a mixture of $2 \mathrm{~mL} / \mathrm{kg} 50 \%$ zoletil and 50\% xylazine hydrochloride and perfused through the heart with $200 \mathrm{~mL}$ of $0.9 \% \mathrm{NaCl}$ solution, followed by $4 \%$ paraformaldehyde solution. The obtained samples were embedded in paraffin and sectioned at a thickness of $10 \mu \mathrm{m}$ for immunohistochemistry and H-E staining. In brief, the sections were washed $(3 \times 10 \mathrm{~min})$ in $0.01 \mathrm{M}$ phosphate-buffered saline solution (PBS; $\mathrm{pH}$ 7.2) and incubated with appropriate primary antibodies for $12 \mathrm{~h}$ at room temperature. The antibody was diluted to 1:200 with a solution of Triton X-100 and normal donkey serum. After incubation in primary antibody, the sections were rinsed $(3 \times 10 \mathrm{~min})$ in PBS, incubated for $90 \mathrm{~min}$ at room temperature with anti-mouse immunoglobulin (Ig) G (Vector Laboratories Inc, USA), diluted 1:25 in a solution of Triton X-100 and normal donkey serum. After incubation in secondary antibody, the sections were rinsed $(3 \times 10 \mathrm{~min})$ in PBS, and incubated for 1 hour at room temperature with Vectastain Elite ABC-kit (Vector Laboratories Inc, USA). Then, the sections were rinsed again with PBS and incubated for 10 minutes in $0.04 \mathrm{mg}$ of 3,3'-diaminobenzidine (DAB) in $200 \mathrm{~mL}$ distilled water. The sections were then incubated for 1 minute in DAB solution with $35 \% \mathrm{H}_{2} \mathrm{O}_{2}$. The DAB sections were rinsed again with $\mathrm{PBS}(3 \times 10 \mathrm{~min})$ to halt the chromagen reaction, wet-mounted on gelatin/chromiumcoated slides, and allowed to air-dry overnight. The sections were then dehydrated through a series of alcohols, soaked in xylene, and cover-slipped with Clarion (Biomedia, USA). To confirm the histological changes in brain tissue, the sections were washed $(3 \times 10 \mathrm{~min})$ in $0.01 \mathrm{M}$ phosphate-buffered saline (PBS, $\mathrm{pH}$ 7.2). Then, they were dipped in hematoxylin solution for 5 minutes and washed under running tap water. The sections were dipped in 1\% $\mathrm{HCl}$-alcohol solution 4 times, then, washed in running tap water for 5 minutes. After washing, they were dipped in eosin solution for 2 minutes, dehydrated via a graded series of alcohols, soaked in xylene and cover-slipped with Clarion (Biomedia, USA). Western blotting analysis was performed for quantitative investigation of the protein expression in the muscle tissue. Muscle samples from each group of rats were collected, washed twice in PBS, and then homogenized and lysated with buffer $\left(137 \mathrm{mM} \mathrm{NaCl}, 8.1 \mathrm{mM} \mathrm{Na} \mathrm{HPO}_{4}, 2.7 \mathrm{mM} \mathrm{KCl}, 1.5 \mathrm{mM}\right.$ $\mathrm{KH}_{2} \mathrm{PO}_{4}, 2.5 \mathrm{mM}$ EDTA, $1 \mathrm{mM}$ dithiothreitol, $0.1 \mathrm{mM}$ $\mathrm{PMSF}$, and $10 \mu \mathrm{g} / \mathrm{ml}$ leupeptin [pH 7.5]) for 30 minutes on ice. Then, the lysates were centrifuged for 10 minutes at $15,000 \mathrm{rpm}$ at $4{ }^{\circ} \mathrm{C}$, and the protein concentration was determined, as described previously 12 . Equal amounts of protein (i.e. $40 \mu \mathrm{g}$ ) were resolved via $10 \%$ sodium dodecyl sulfatepolyacrylamide gel electrophoresis (SDS-PAGE) and transferred to nitrocellulose membranes. The blots were then washed with TBST (10 mM Tris $\cdot \mathrm{HCl}[\mathrm{pH} 7.6], 150 \mathrm{mM}$ $\mathrm{NaCl}, 0.05 \%$ Tween 20 ), blocked with $5 \%$ skim milk for 1 hour, then incubated with the appropriate primary antibodies at the dilutions recommended by the manufacturers. The membranes were washed, and the primary antibodies were detected using horseradish peroxidase-conjugated goat antirabbit IgG or goat-anti mouse IgG. The bands were then visualized via enhanced chemiluminescence (Amersham Pharmacia Biotech, Piscataway, NJ, USA). The results are expressed as mean \pm standard error (S.E.). All results were analyzed with analysis of variance (ANOVA), and comparisons of the mean values between the treatment and control groups were made using the Bonferroni-Dunn test. Differences were considered statistically significant at values of $\mathrm{p}<0.05$. All analyses were performed using SPSS for Windows (v. $12.0 \mathrm{~K}$, SPSS Inc., USA).

\section{RESULTS}

Immunoblotting of HSP 70 expression was performed for each group. The optical density values relative to the CON group values on day 1 after injury were compared between groups and time points. The expression of HSP 70 tended to increase significantly with lapse of time in the PEMF and DPEMF groups, however, it decreased in CON $(p<0.05)$. At 1 day after injury, there were not significant differences among the three groups ( $>0.05)$, though there was a small increase in the PEMF group. At 3 days after injury, a more significant increase in HSP 70 expression presented in the PEMF group than in the DPEMF group $(\mathrm{p}<0.05)$. There were significant increases in the PEMF and DPEMF groups 5 days after injury with a greater increase in the PEMF group $(p<0.05)$ (Table 1A). The immunohistochemistry results of HSP 70 expression were similar to those of immunoblotting at 5 days after injury (Table 1B).

To observe the histological changes induced by crush injury and therapeutic application, hematoxylin-eosin (H-E) staining was carried out 5 days after injury. General severe atrophy of muscle fiber and irregular arrangement was seen in the CON group. However, muscle atrophy and arrangement were improved in the PEMF and DPEMF groups with greater recovery in the PEMF group (Fig. 1).

\section{DISCUSSION}

The principal clinical indications for PEMF are fracture healing, degenerative diseases of the musculoskeletal system, disorders of the neurological system, muscle spasm, 
Table 1A. The effect of pulsed electromagnetic field on the expression of HSP 70 after muscle crush injury

\begin{tabular}{lccc}
\hline \multirow{2}{*}{ Group } & \multicolumn{3}{c}{ Relative optical density (\% of CON of on day 1 after injury) } \\
\cline { 2 - 4 } & 1 day & 3 days & 5 days \\
\hline CON & $99.6 \pm 1.4$ & $84.6 \pm 1.6^{*}$ & $65.2 \pm 1.4^{*}$ \\
PEMF & $103.9 \pm 1.5$ & $134.1 \pm 3.4 * \S$ & $141.4 \pm 4.4 * \S$ \\
DPEMF & $96.2 \pm 1.7$ & $104.3 \pm 2.6 \S$ & $115.6 \pm 2.4 * \S$ \\
\hline
\end{tabular}

Table 1B.

\begin{tabular}{cccc}
\hline \multirow{2}{*}{ Group } & \multicolumn{3}{c}{ Relative optical density (\% of CON of on day 5 after injury) } \\
\cline { 2 - 4 } & CON & PEMF & DPEMF \\
\hline 5 days & $99.4 \pm 1.0$ & $141.8 \pm 3.4^{*}$ & $113.3 \pm 2.1^{*}$ \\
\hline
\end{tabular}

To confirm the expression of HSP 70, western blotting (A) and immunohistochemistry (B) were conducted on samples from the CON, PEMF, and DPEMF groups. Each example shown is representative of three experiments. The optical density values are the mean \pm S.E. of the values of relative to the CON group on days 1 and 5 after injury. They were determined by densitometry relative to $\beta$-actin. Statistical analysis was performed by using two-way ANOVA. $* p<0.05$ versus day 1 after injury between the period; $\S p<0.05$ versus $\mathrm{CON}$ between the group

\section{CON}

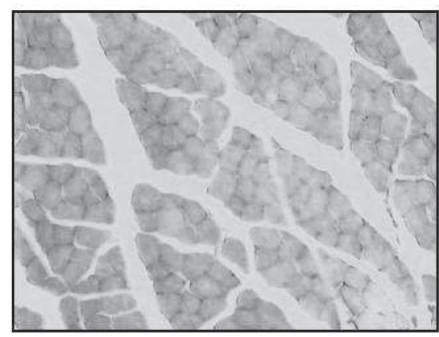

PEMF

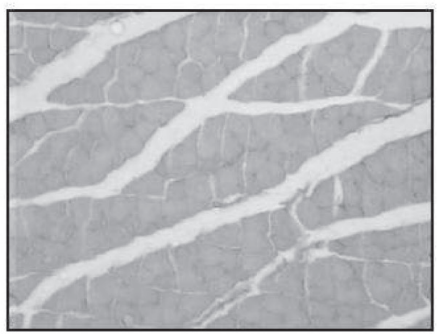

DPEMF

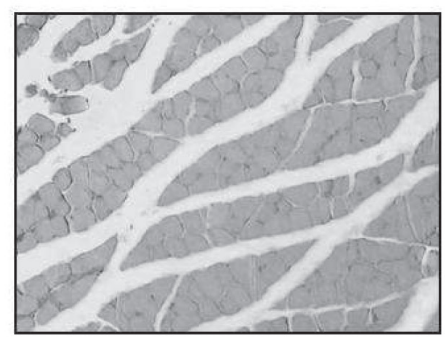

Fig. 1. The effect of pulsed electromagnetic field after muscle crush injury from H-E staining. To confirm the histological change, H-E staining was conducted on samples the CON, IST, and CST groups $(\times 100)$.

generalized inflammation, and poor circulation therapeutic properties of PEMF are well known, however, studies of interactions between organic tissues and PEMFs are few 13 . The purpose of present study was to confirm the effects of electromagnetic fields applied at different time points on muscle recovery through the observation of the expression of HSP 70 protein and histological changes in a rat muscle crush injury model.

It has been reported that the synthesis of HSP 70, which can be defined as HSP 70 expression, tends to be elevated in the acute phase of tissue injury. HSP, a stress protein, is synthesized shortly after cells are exposed to stress or a rapid rise in temperature 14 . It functions to minimize cell injury by maintaining proper protein folding with degenerative enzyme proteins and by supporting intracellular homeostasis, including protein transport and synthesis 1,15 . Although HSP 70 is spontaneously expressed under rapidonset pathological conditions, it does not sufficiently protect against cell injury.
In the present study, the application of PEMF increased the expression of HSP 70 protein in injured muscle which also showed histological improvement. A previous study reported that PEMF induced the cellular heat shock factor (HSF) 1 response, and released HSP 90 from the heat shock transcription factor 1 in primary human $\mathrm{T}$ lymphocytes and fibroblast cell lines ${ }^{10}$. PEMF increased HSF1 phosphorylation and HSF1-DNA binding together; these factors improve HSP 70 expression. Goodman et al. ${ }^{17}$ demonstrated that magnetic field-exposed cells exhibited HSF1 DNA-binding activity and heat shock element-binding in a sequence of events that mediated the transcription of the HSP 70 gene and synthesis of the HSP 70 protein. Moreover, George et al. 18 reported that electromagnetic field induction of HSP 70 improved myocardial function. These results indicate that PEMF application is beneficial for the prevention of the pathological progression of muscle injury and the promotion of the repair mechanism by maintaining HSP 70 expression. 
The healing process of the muscle generally includes three continuous processes of destruction, repair, and remodeling 1 . In the primary phase, the rupture and necrosis of myofibers and hematoma formation occurs in the injured portion, and chemokines, growth factors, and cytokines are required by inflammatory cells for recovery 19,20 . This phase induces and overlaps with the repair phase. In addition, muscle healing involves the formation of connective scar tissue, not primarily regeneration. The healing process of injured muscle can frequently be hampered by the formation of scar tissue which is characterized by extensive muscle

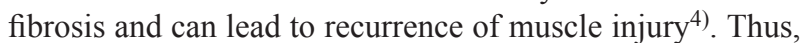
a therapeutic approach in this phase is important to decrease scar tissue and promote the alignment of muscle fiber for normal contraction. Our results show there was a the more significant increase in HSP 70 expression and greater histological improvement in the PEMF group than in the DPEMF group. This suggests that application of PEMF is helpful in the treatment of muscle repair.

Our results suggest that PEMF enhances the recovery of injured muscle via HSP 70 expression. More study will be needed to establish the clinical aspects for humans. The present study provides useful evidence of the biological effects of PEMF, which might exert a positive effect on intracellular metabolism stimulate metabolic activation in the extracellular matrix. It will be necessary to perform experiments in various injury models and environments to provide data for evidence-based practice.

\section{ACKNOWLEDGEMENT}

This research was supported by Youngsan University Research Grants in 2011 .

\section{REFERENCES}

1) Järvinen TA, Jarvinen TL, Kaariainen M, et al.: Muscle injuries: biology and treatment. Am J Sports Med, 2005, 33: 745-764. [Medline] CrossRef

2) Criswell TL, Corona BT, Ward CL, et al.: Compression-induced muscle injury in rats that mimics compartment syndrome in humans. Am J Pathol,
2011, 180: 787-797.

3) Stratos I, Li Z, Rotter R, et al.: Inhibition of caspase mediated apoptosis restores muscle function after crush injury in rat skeletal muscle. Apoptosis, 2011, 17: 269-277.

4) Rotter R, Menshykova M, Winkler $T$, et al.: Erythropoietin improves functional and histological recovery of traumatized skeletal muscle tissue. J Orthop Res, 2008, 26: 1618-1626. Medline] CrossRef

5) Markov MS: Expanding use of pulsed electromagnetic field therapies. Electromagn Biol Med, 2007, 26: 257-274. [Medline [CrossRef

6) Hug K, Roosli M: Therapeutic effects of whole-body devices applying pulsed electromagnetic fields (PEMF): A systematic literature review. Bioelectromagnetics, 2011, 33: 98-105. [Medline]

7) Canapp DA: Select modalities. Clin Tech Small Anim Pract, 2007, 22: 160-165. [Medline [CrossRef

8) Bassett CA: Fundamental and practical aspects of therapeutic uses of pulsed electromagnetic fields (PEMFs). Crit Rev Biomed Eng, 1989, 17: 451-529. Medline

9) Gupta A, Taly AB, Srivastava A, et al.: Efficacy of pulsed electromagnetic field therapy in healing of pressure ulcers: A randomized control trial. Neurol India, 2009, 57: 622-626. [Medline] [CrossRef]

10) Jasti AC, Wetzel BJ, Aviles H, et al.: Effect of a wound healing electromagnetic field on inflammatory cytokine gene expression in rats. Biomed Sci Instrum, 2001, 37: 209-214. Medline

11) Sutbeyaz ST, Sezer N, Koseoglu F, et al.: Low-frequency pulsed electromagnetic field therapy in fibromyalgia: a randomized, double-blind, sham-controlled clinical study. Clin J Pain, 2009, 25: 722-728. Medline CrossRef

12) Bradford MM: A rapid and sensitive method for the quantitation of microgram quantities of protein utilizing the principle of protein-dye binding. Anal Biochem, 1976, 72: 248-254. Medline] CrossRef]

13) Gómez-Ochoa I, Gomez-Ochoa P, Gomez-Casal F, et al.: Pulsed electromagnetic fields decrease proinflammatory cytokine secretion (IL-1beta and TNF-alpha) on human fibroblast-like cell culture. Rheumatol Int, 2011, 31: 1283-1289. Medline CrossRef

14) Ellis RJ, van der Vies SM: Molecular chaperones. Annu Rev Biochem, 1991, 60: 321-347. Medline CrossRef

15) Lancaster GI, Febbraio MA: Exosome-dependent trafficking of HSP70: a novel secretory pathway for cellular stress proteins. J Biol Chem, 2005, 280: 23349-23355. [Medline CrossRef

16) Perez FP, Zhou X, Morisaki J, et al.: Electromagnetic field therapy delays cellular senescence and death by enhancement of the heat shock response. Exp Gerontol, 2008, 43: 307-316. Medline [CrossRef

17) Goodman R, Blank M: Magnetic field stress induces expression of hsp70. Cell Stress Chaperones, 1998, 3: 79-88. [Medline [CrossRef

18) George I, Geddis MS, Lill Z, et al.: Myocardial function improved by electromagnetic field induction of stress protein hsp70. J Cell Physiol, 2008, 216: 816-823. Medline [CrossRef

19) Huard J, Li Y, Fu FH: Muscle injuries and repair: current trends in research. J Bone Joint Surg Am, 2002, 84-A: 822-832. [Medline]

20) Chargé SB, Rudnicki MA: Cellular and molecular regulation of muscle regeneration. Physiol Rev, 2004, 84: 209-238. [Medline] [CrossRef] 